\title{
Multiparticle Simulation of Ion Motion in the Ion Trap Mass Spectrometer: Resonant and Direct Current Pulse Excitation
}

\author{
R. K. Julian,* M. Nappi, ${ }^{\dagger}$ C. Weil, ${ }^{\dagger}$ and R. G. Cooks \\ Department of Chemıstry, Purdue University West Lafayette, Indiana, USA
}

\begin{abstract}
A PC-based program that simulates the behavior of a collection of ions is extended to include the effects of collisions with the buffer gas and enhanced visualization methods. The simulations are based on the quadrupole field associated with the actual ion trap electrode structure. Ionization is simulated in such a way as to distribute ionization events randomly over rf phase angles and yield a realistic collection of stored ions. The effects of buffer gas collısions on ion motion during both mass-selective instability and resonance ejection scans are found to include the expected dampening of spatial excursions as well as limitation of the kinetic energy of trapped ions. In both experıments, ion ejection occurs over a number of secular cycles in the vicinity of the theoretical instability point. Activation via a resonant ac signal or a short dc pulse is shown to result in phase-locking of the ions as well as the expected increase in the size of the excursions in the $z$ direction and in ion kinetic energy. Collisions cause dephasing and loss of kinetic energy. Radıal dc activation is compared with activation in the axial direction. Experimental data for dc pulse activation of the $n$-butylbenzene molecular ion are analyzed in phase space and the onset of surface-induced dissociation is correlated with changes in the experimental $\mathrm{m} / z$ 91 to $\mathrm{m} / z$ 92 fragment ion ratio. Poincaré sections are shown for resonantly excited lons and their value in demonstrating improvement of the resolution of these experiments over conventional mass-selective instability scans is shown. ((j Am Soc Mass Spectrom 1995, 6, 57-70)
\end{abstract}

W 1th the growing importance of the quadrupole ion trap as an analytical mass spectrometer $[1,2]$, interest has increased in simulating ion motion within the device. In a series of papers [3-5] March and co-workers demonstrated the value of single ion simulations. Their simulations included ion motion during resonant excitation and showed that several frequencies that are not present normally as components of ion motion are present during dipolar and quadrupolar excitation. They also described the energy required to excite ions to a particular kinetic energy level as a fluence, which can be used as a standard measurement of excitation, that incorporates both excitation voltage and time. In spite of these successes, there are limitations in single ion simulations, including lack of access to ion-ion interactions.

We have developed two simulation programs to examine the behavior of collections of ions in the ion trap: NQS was designed for parallel computers [6],

Address reprint requests to Professor R Graham Cooks, Department of Chemistry, Purdue Unuversity, 1393 Brown Building, Rec Room 2494, West Lafayette, IN 47907

* Present address Lilly Research Laboratones, Indianapolis, Indiana ${ }^{\dagger}$ On leave from Physikalısches Institut, Unıversitat Giessen, West Germany whereas ITSIM handles fewer ions, but runs on PC machines [7]. In the present study, ITSIM is extended to include the effects of collisions with buffer gas.

The primary goal of modeling the behavior of a limited number of ions (less than 1000) in a quadrupole ion trap is visualization of ensembles of ions to give insight into the character of trajectories (i.e., frequency, phase relationshup, and magnitude) and to allow a direct comparison between trajectories of lons with different mass-to-charge ratio values. Phase-space plots of single particles have proven to be a valuable tool in analyzing the dynamics of the ion trap for some time $[8,9]$. A plot that shows the phase-space dynamics of several particles adds the ability to determine the ranges of position and kinetic energy withen the ion cloud as a function of time under various operating conditions. Memory limits for DOS-based $80 \times 86$ PC machines set the limit for the number of ions that may be simulated with the PC-based ITSIM program [10], whereas speed considerations limit the total simulation time over which the trajectories may be accurately integrated.

In this article, multiparticle simulations are used to characterize the motion of ensembles of ions under various kinetically excited conditions. These conditions include (1) ejection via the $\beta_{z}=1$ boundary, the 
mass-selective instability scan [11], (2) resonant ejection and resonant excitation via an auxiliary (ac) field [12], and (3) dc pulse activation [13-15]. The spatial and temporal relationships between ions of the same mass-to-charge ratio under kinetically excited conditions are important because of the consequences for the mass resolution of the ejected ions. Similarly, the amount of energy deposited via excitation from an ac field or a dc pulse depends on these relationships. The key to mapping ion behavior is a description of the motion of the ions within the ion trap under the influence of collisions. Attention is therefore given to buffer gas collisions, which are treated as random discrete events with different impact parameters. Buffer gas collisions are expected to have a large effect on the nature of ion motion in that ions that have similar amplitude and frequency and are in phase with one another will tend to de-phase and lose kinetic energy when buffer gas is present. Collisions also allow ions to remain within the dimensions of the ion trap electrodes, despite the mathematical instability of a simple Mathieu description of its trajectory. A hard-sphere model is employed in which the scattering angle is zero, a reasonable approximation for the heavy projectile-light target combination used here. Note that in addition to the particular collision model for which results are described here, a number of related models have been examined. Although there are differences in detail, the overall character of ion motion is reproduced in these simulations. Note also that the data shown in this article represent results for particular operating conditions and that small changes in operating conditions can result in ion motion with substantially different character, especially in resonance experiments.

The Poincare section is used, together with phasespace and displacement versus time plots, to capture the information in the simulations.

\section{Equations of Motion}

The factors that control ion motion in a quadrupole electric field can be expressed as a second-order dif- ferential equation that describes the acceleration of an ion:

$$
\begin{aligned}
\frac{d^{2} z}{d t^{2}}= & -C(t) \frac{d z}{d t} \\
& +\frac{4 e}{m\left(r_{0}^{2}+2 z_{0}^{2}\right)}(U(t)-V(t) \cos \Omega(t) t) z \\
& +\frac{e}{m 2 z_{0}} V_{\text {aux }}(t) \cos \Omega_{\text {aux }}(t) t \\
& +\frac{e^{2}}{4 \pi \varepsilon_{0} m} \sum_{t} \frac{1}{\left(r-r_{1}\right)}
\end{aligned}
$$

where $z$ is given as the axis of motion. Similar equations can be written for the $x$ and $y$ directions and all three dimensions were considered in the simulations. Other parameters include the following: $t$ is time, $m$ is defined as the mass-to-charge ratio for simplicity, $e$ is the charge on a single electron, $\varepsilon_{0}$ is the permittivity of vacuum, and $C(t)$ is a time-dependent energy loss term that will be discussed further. $V(t), \Omega(t), V_{\text {aux }}(t)$, $\Omega_{\mathrm{aux}}(t)$, and $U(t)$ are time-dependent operating parameters given in Table 1 , and $r_{0}$ and $z_{0}$ are geometric parameters given in Table 2. Note that the second right-hand side term expresses forces due to the quadrupole field and the third term expresses those due to the auxiliary dipole field used for resonance excitation. Space-charge is considered in the fourth term as a Coulombic repulsive potential. In this term, $r$, represents the positions of all other ions. Note that the space-charge term involves all three coordinates with $\mathbf{r}=(x, y, z)$, coupling the motion of ions in all three directions. As usual, radial symmetry obtains, which allows representation of motion by displaying trajectories in the $z$ and $x$ directions only. The simulations shown here are for small numbers of ions for which the computationally intensive space-charge term was shown to have no effect and is therefore not utilized.

The term $C(t)$, as stated in eq 1 , is normally treated in simulation codes as a constant velocity reduction

\begin{tabular}{|c|c|c|c|}
\hline $\begin{array}{l}\text { Operating } \\
\text { parameter }\end{array}$ & $\begin{array}{l}\text { Parameter in } \\
\text { equations } \\
\text { of motion }\end{array}$ & Units & $\begin{array}{c}\text { Parameter } \\
\text { update criteria }\end{array}$ \\
\hline $\begin{array}{l}\text { if Amplitude } \\
\text { if Frequency }\end{array}$ & $\begin{array}{l}V \\
\Omega\end{array}$ & $\begin{array}{l}V_{0-p} \\
H z\end{array}$ & $\begin{array}{l}\text { Update only at if zero crossıngs } \\
\text { Update only at if phase mınıma } \\
\text { or maxıma }\end{array}$ \\
\hline $\begin{array}{l}\text { ac Amplitude } \\
\text { ac Frequency }\end{array}$ & $\begin{array}{l}V_{\text {aux }} \\
\Omega_{\text {aux }}\end{array}$ & $\begin{array}{l}\mathrm{V}_{0-0} \\
\mathrm{~Hz}\end{array}$ & $\begin{array}{l}\text { Update only at ac zero crossıngs } \\
\text { Update only at ac phase mınıma } \\
\text { or maxıma }\end{array}$ \\
\hline dc Amplitude & $U$ & $V_{d c}$ & Update every clock tıck \\
\hline
\end{tabular}

Table 1. Operating parameters for the quadrupole ion trap mass spectrometer and the related variables in the equation of motion as well as the criteria for updating the value of the parameter by the scheduler routine 
Table 2. Geometric parameters used to describe quadrupole ion trap electrodes ${ }^{\mathrm{a}}$

\begin{tabular}{ccccc} 
Geometrıc & $\begin{array}{c}\text { Simplified } \\
\text { geometry } \\
r_{0}^{2}=2 z_{0}^{2}\end{array}$ & $\begin{array}{c}\text { ITMS } \\
\text { geometry }\end{array}$ & $\begin{array}{c}\text { ITMS dimension } \\
\text { measurement } \\
\text { error }\end{array}$ & Geometrıc definition \\
\hline$r_{0}$ & 10000 & 1000 & 0001 & $\begin{array}{c}\text { Radius of the } \text { ring } \\
\text { electrode }(\mathrm{cm}) \\
\text { Inscribed radius of the endcap } \\
\text { electrode }(\mathrm{cm})\end{array}$ \\
\hline
\end{tabular}

${ }^{8}$ Measured from actual electrode The values for $r_{0}$ and $z_{0}$ describe the size of the electrodes

taken over the integration period used to solve eq 1 . This method ignores the random nature of the collision process and the effects of losing varying amounts of energy through collisions at a variety of ion positions and velocities. Simple viscous drag models of ion-neutral collisions also preclude the consideration of a velocity dependence in the collision probability. Although there is evidence [16] that at low ion energy the velocity-independent Langevin model can accurately describe some aspects of ion motion, at higher energies hard-sphere collisions are expected to play a more important role in ion energy loss, and this is the form of collision process modeled here.

Equation 1 implements the general form of the quadrupole potential given by Knight [17]. The purpose for this generalization is to account for the change in the quadrupole field strength due to the stretched geometry of the ITMS instrument [18]. The parameter $q_{z}$, used to express the operating point for a given ion, can be expressed in the general form, which allows it to be computed for a given set of operatung conditions.

$$
q_{z}=-2 q_{r}=\frac{-8 e V}{m\left(r_{0}^{2}+2 z_{0}^{2}\right) \Omega^{2}}
$$

In the simulation, eq 1 is solved by using a Taylor series expansion in two steps: one for velocity and one for position. First the velocity term is updated,

$$
\begin{aligned}
\frac{d u}{d t}\left(t_{n+1}\right)= & \frac{d u}{d t}\left(t_{n}\right)+\Delta t \frac{d}{d t}\left(\frac{d u}{d t}\right)\left(t_{n}\right) \\
& +\cdots+\frac{(\Delta t)^{s}}{s !} \frac{d^{s}}{d t}\left(\frac{d u}{d t}\right)\left(t_{n}\right)
\end{aligned}
$$

and then the position term is updated,

$$
\begin{aligned}
u\left(t_{n+1}\right)= & u\left(t_{n}\right)+\Delta t \frac{d u}{d t}\left(t_{n}\right) \\
& +\frac{(\Delta t)^{2}}{2 !} \frac{d^{2} u}{d t^{2}}\left(t_{n}\right)+\cdots+\frac{(\Delta t)^{s}}{s !} \frac{d^{s} u}{d t^{s}}\left(t_{n}\right)
\end{aligned}
$$

where $u$ is the position in the $x, y$ and $z$ directions.

The $C(t)$ term in eq 1 is implemented in the code by scaling the velocity term by using a collisional damp- ing term $\Lambda(t)$,

$$
\frac{d u}{d t}\left(t_{n+1}\right)=\Lambda\left(t_{n}\right) \frac{d u}{d t}\left(t_{n}\right)
$$

where $\Lambda\left(t_{n}\right)$ is a quantity varying from 1 (flyby) to the maximum amount of velocity loss possible in a head-on collision. To obtain $\Lambda\left(t_{n}\right)$ we split the collision effects into two parts, one of which determines if a discrete collision occurs in the current time step, and the other that reduces the current velocity $v(t)$ of the ion by an amount that varies with the hard-sphere impact parameter. The probability of the occurrence of a collision is $P(t)$

$$
P(t)=\nu(t) d t=\frac{\sqrt{v_{\mathrm{r}}(t)^{2}+v_{y}(t)^{2}+v_{z}(t)^{2}}}{\lambda_{m}} d t
$$

where $\nu(t)$ is the collision frequency and $d t$ is the integration time step size, and where

$$
\frac{1}{\lambda_{m}}=N\left(\pi D_{12}^{2}\right)=N \sigma
$$

Equation 7 gives the mean-free path $\lambda_{m}$ in terms of the collision cross section $\sigma$, which is defined here in terms of the ion and neutral diameters $D_{1}$ and $D_{2}$, namely, $D_{12}=\left(D_{1}+D_{2}\right) / 2$. The number density of the buffer gas $(N)$, is used to account for the buffer gas pressure. A binary function $L(t)$ is therefore defined as 1 when a collision occurs and 0 when no collision occurs during the current time interval, namely,

$$
L(t)= \begin{cases}1 & \text { for } R_{1}(t)>P(t) \\ 0 & \text { for } R_{1}(t) \leq P(t)\end{cases}
$$

where $R_{1}(t)$ is a random number from 0 to 1 . By a straightforward derivation based on the fractional velocity lost in an elastic collision [19], the velocity scaling function $\Lambda(t)$ can be written

$$
\Lambda(t)=1-\left[\left(1-\left\{1-\frac{4 m_{b}}{m_{1}}\right\} 1 / 2\right) R_{2}(t)\right] L(t)
$$


where $m_{1}$ is the mass of the ion, $m_{\mathrm{b}}$ is the mass of the buffer gas, and $R_{2}(t)$ is a second random number ranging between 0 and 1 that represents an impact parameter. Note that the velocity-dampening term is scaled inversely with the magnitude of the impact parameter. The value $L(t)$ is either 1 or 0 exclusively and it represents the discrete nature of the collision event. Note that the formulation of the collision process given in eqs 5-9 contains several approximations, the most important of which is that the ions are much more massive than the target, the collisions are hard sphere, and they lead to zero angle scattering. The more complex case of nonzero angle scattering will be implemented in future work.

It is easy to keep track of the number of collisions undergone by each ion in the simulation and hence distributions of collision numbers can be displayed. In cases in which no ions are lost during a simulation, the mean collision frequency is given by the mean number of collisions divided by the simulation period. Typical collision frequencies for populations of trapped ions depend strongly on the initial spatial distribution and Mathieu operating parameter, but values in the tens of kilohertz are common.

The present set of results was obtained under a uniform set of conditions with one particular collision model. The only conditions that normally are varied are the initial distribution of the ion cloud, the pressure, and the mass-to-charge ratio value of the ion. All voltages are zero-to-peak values. The con and buffer gas diameters are taken as 10 and $1 \AA$, respectively. The simulations all refer to the stretched geometry of the commercial ion trap. Higher order components of the electric field are not considered.

\section{Simulation of Ionization-Effects of Initial rf Phase}

Ions with identical mass-to-charge ratios have trajectories that contain the same frequency components. The trajectories are identical except for a scaling factor that corresponds to the maximum displacement possible for the initial position and velocity. The basic nature of this scaling can be observed in Figure 1a. In this simulation, the trajectories for 10 ions of $m / z 202$ are computed at an rf ring voltage of $1110 \mathrm{~V}_{0-p}$ $\left(q_{z}=0.4\right)$. This simulation shows the consequences of specifying the initial conditions of the ions with no allowance for differences in the initial rf phase. The zero cross points observed in both the $x$ and $z$ directions are artifacts of the identical initial rf phase of each ion. The dephasing effects due to buffer collisions can be observed as a smear in the zero crossing later in time when the simulation is done considering the effect of buffer gas (not shown). The apparent phaselocking of the ions to each other precludes studies in which the phase relationships between ions of like mass-to-charge ratio acquired during excitation are examined. There is no reason to expect the ions to be in phase as a result of the ionization process; therefore a correction to this initial condition problem was sought.

To achieve this correction, a model of the ionsation event was introduced into ITSIM. In the ionization simulation, known as IONGEN, ions are inutially marked as neutral and no trajectory is computed until Ionization is initiated at a random time during the 1onization period. An example of such an ionization simulation is given in Figure $1 b$, which shows the results of repetition of the storage simulation from Figure 1a, after the initial rf phase of the ions are randomly distributed by the ionization component of the simulation. No phase coherence is observed in the secular motion and this allows the evaluation of phase coherence produced by various excitation techniques. Because each ion begins at a random rf phase, some ions fall outside the phase-space acceptance ellipse and are not trapped during the ionization period. During an actual experiment, more molecules are ionized, perhaps with a wider range of intial positions, which leads to large numbers of lost ions. The simulation deliberately shows the behavior of a small number of ions. When the simulation is repeated with a larger number of ions, averaging of positions and velocities makes the features of interest difficult to display.

The buffer gas plays an important role during ionization by dampening the ion motion, which thereby compresses the ion cloud in phase space to fall within the acceptance limits of the ion trap. The result, for both the electron ionuzation experiment and ion injection experiments, is that more ions are trapped. This is the cause of the well known improvement in sensitivity and signal-to-noise ratio observed when a buffer gas is used [11].

\section{Effects of Buffer Gas Collisions on Ion Ensembles}

Five ion trap experiments were examined by using simulations of the behavior of collections of ions under the influence of collisions. These experiments are (1) simple ion trapping under fixed conditions (2) the mass-selective instability scan, conventionally used to record mass spectra, (3) the resonance ejection experiment, which is used to enhance resolution and to extend the mass range of the instrument, (4) resonance activation, used for collision-induced dissociation, and (5) a new experiment that involves the application of dc pulses to the endcap electrodes in an attempt to force the ions further from the center or to force collisions with electrode surfaces [13]. Because collections of ions are observed in each of these simulations, a flow [6], along a solution surface can be sampled instead of a single path on that surface, and this gives information on ranges of such values as energy, position, and ejection conditions. 

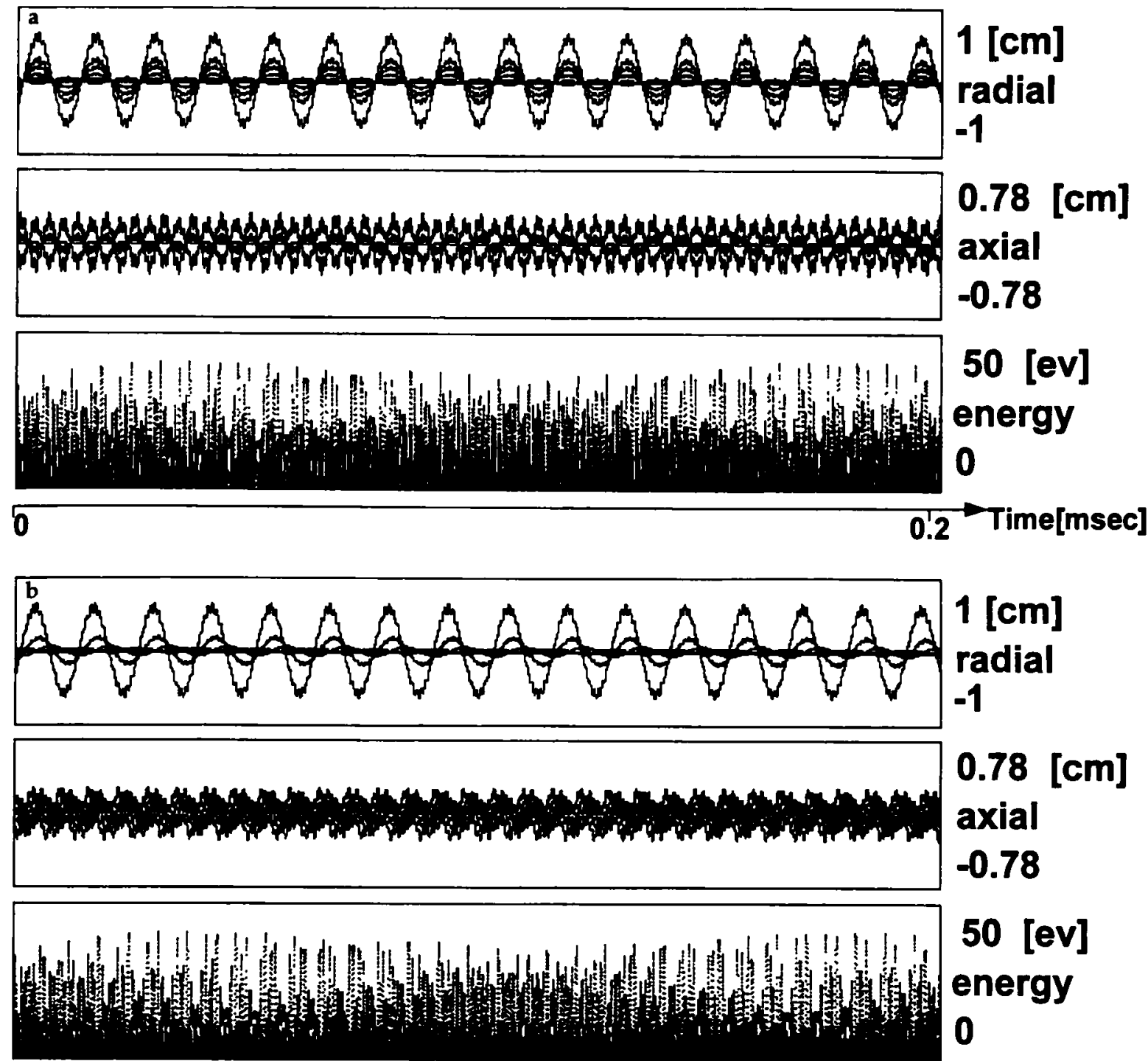

50 [ev] energy 0

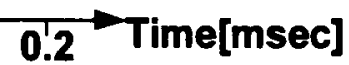

Figure 1. Ion storage simulation for 10 ions of $m / z 202$ with an initial Gaussian distribution (FWHM $7 \mathrm{~mm}$ ) are stored at $q_{z}=04$ Ions are distributed in position and velocity, but have identical initial rf phase angles. Note that the individual ion trajectories differ only by a scaling factor that corresponds to different maximum amplitudes Trajectory and energy plot when (a) ions have identical initial rf phases and (b) initial phases are randomized

\section{Trapped Ions}

Effects of collisions were examined for a collection of 200 ions, $m / z 105$, randomly distributed in phase and with an intial spherically symmetrical Gaussian distribution [full width at half-maximum (FWHM) $5 \mathrm{~mm}$ ]. The trapping conditions $q_{z}=0.21$ were held constant and the effects of various buffer gas pressures were examined. Figure 2 shows the results for 20 ions at a relatively high pressure ( $8 \mathrm{~m}$ torr). The most important feature of the simulation is the loss of kunetic energy and contraction of the excursions in both the $r$ and $z$ directions that occurs as a result of collisions. Note that the form of the display is such as to give excessive weight to the behavior of outlier ions, namely, those whose energies and trajectories diverge from the average. A second observation, not visible on this display but seen upon magnification, is the stochastic nature of the collisions. Occasional sudden changes in motion are observable in association with collisions. During the $400 \mu \mathrm{s}$ for which this simulation was run, just one ion was lost, early in the period. The collision distribution (Figure $2 b$ ) shows a most probable value of approxımately 30 collisions, which correspond to a collision frequency of $75 \mathrm{kHz}$.

It was of interest to compare the results of this simulation of trapped ions under the influence of collisions at various impact parameters with the results obtained when each collision was assumed to remove the same amount of energy. The average energy loss 

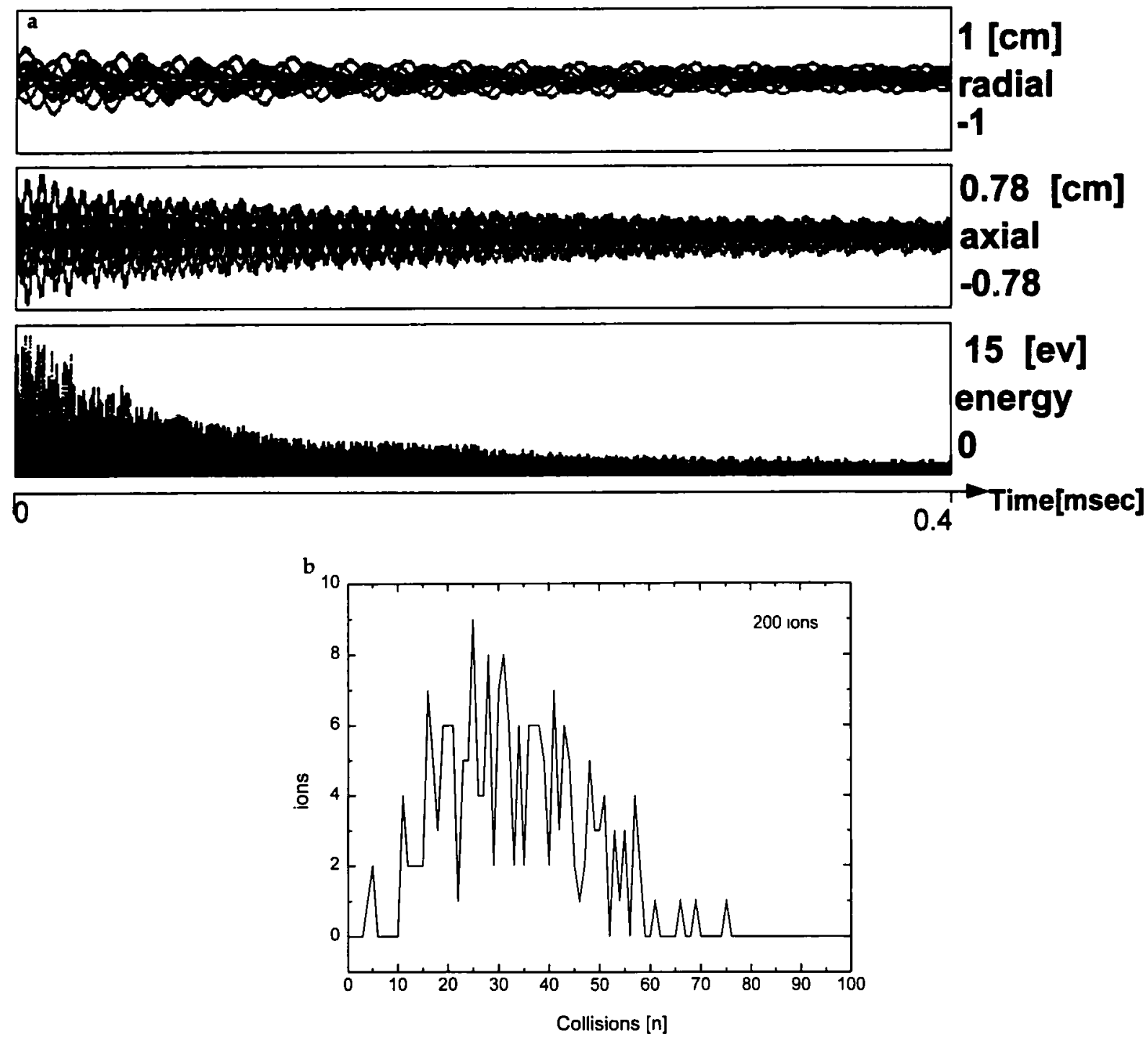

Figure 2. Ion storage sumulation with collisions for cons of $m / z 105$ randomly distributed in initial phase with a symmetrical Gaussian distribution (FWHM $5 \mathrm{~mm}$ ). Data are for 8-mtorr helium buffer gas at $q_{z}=0.21$ (a) Trajectory and kinetic energy plots for 20 1ons, (b) collision distribution for 200 ions.

(over all impact parameters) is known as a function of center-of-mass scattering angle [20]. Simulations based on this simplified model gave similar results to those shown in Figure 2.

\section{Mass-Selective Instability Scan}

The mass-selective instability scan, developed by Stafford and co-workers at Finnigan [11], is one of the most important developments in the field of quadrupole ion trap mass spectrometry. This scan is used to generate mass spectra by mass selectively ejecting rons into an external detector. Later developments, such as the ability to perform multiple stages of mass analysis [21], the extension of the mass range [22], the ability to obtain high resolution spectra [23], and the incredible sensitivity improvements [24], all utilize this scan. It is therefore critical to understand the ion ejection process, which is controlled by the dynamic effects of the scan on ion motion. Figure 3 shows a simulation of ion motion during the massselective instability scan performed in the presence of helium buffer gas. In this simulation, which for the purposes of display uses a relatively high pressure and a very steep ramp of the amplitude of the $\mathrm{rf}$ voltage applied to the ring electrode, ions are forced to acquire mathematically unbounded trajectories. When the amplitude of the ion trajectory exceeds the dimensions of the electrode in the $z$ direction, ions are ejected. Those ions that eject via the exit endcap electrode strike the electron multiplier and are detected. This signal is associated with a particular rf voltage that can be converted to a mass scale to create a mass spectrum. Note in Figure 3 that all ions have the same mass and 


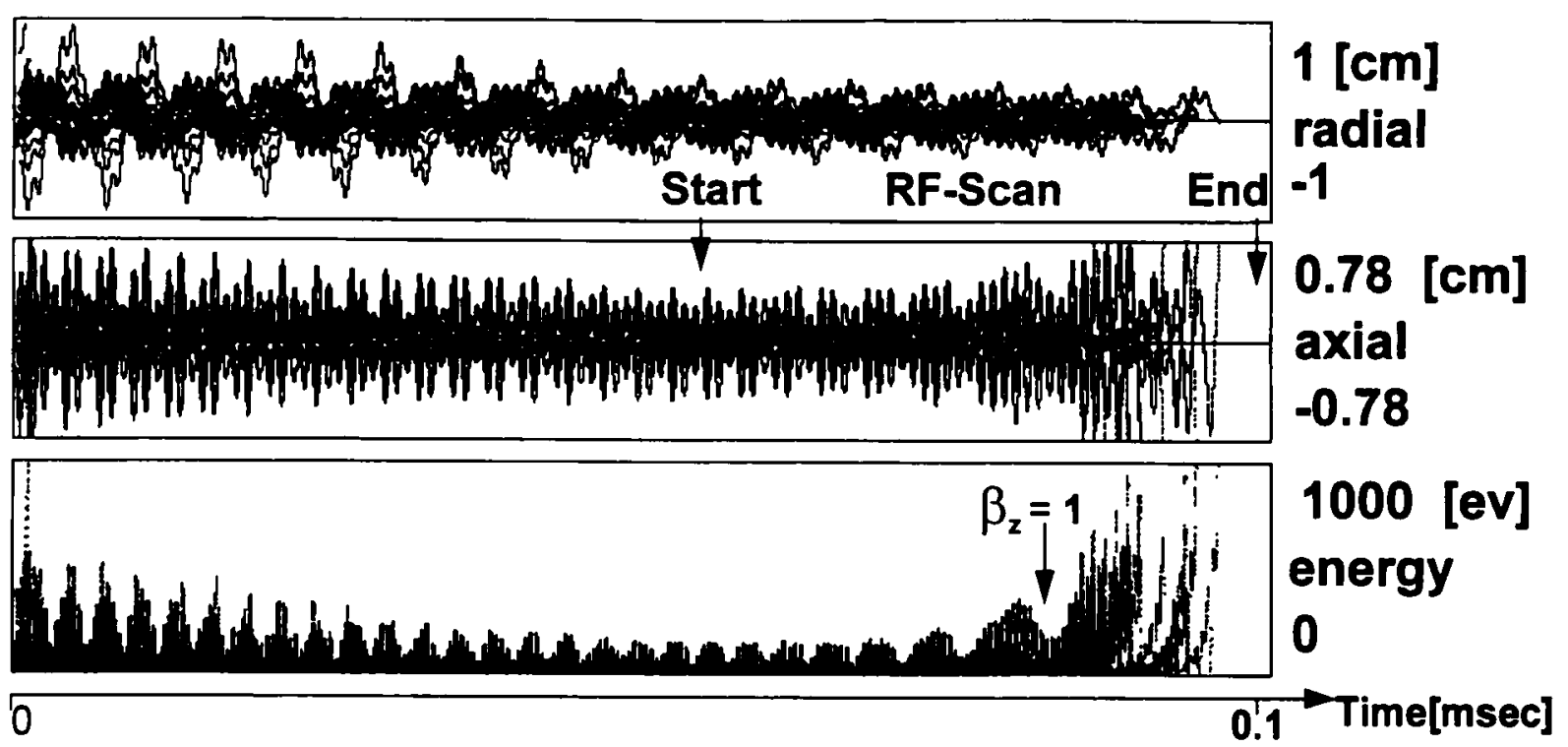

Figure 3. Simulation of mass-selective instability scan (40 1ons of $m / z 202$ trapped at $q_{z}=0.4$, initial distribution as in Figure 2) with helium buffer gas at a partial pressure of 8-mtorr The rf was scanned from 2200 to $2710 \mathrm{v}$ over the interval marked.

hence the same frequency of motion (both the secular frequency and higher frequency components are visible, especially after scale expansion), but are phaseshifted from each other. The rf scan parameters of Figure 3 are chosen to illustrate the fact that buffer gas collisions dampen the amplitude of ion motion and reduce their kinetic energies during the cooling period, whereas the field has the opposite effect during the $\mathrm{rf}$ ramp scan-out period. Note that some ions remain within the electrode boundary despite mathematical instability ( $\beta_{z}$ values greater than unity), in part due to buffer gas collisions. The presence of the buffer gas maintains the ion cloud at a low energy as the stability limit is approached. In separate simulations performed with more realistic of amplitude scan rates and done without buffer gas, the ion cloud is observed to grow steadily in maximum $z$ amplitude as it approaches the stability limit; ions are lost during the cloud expansion. On the other hand, in the presence of buffer gas, the ion cloud diameter is maintained at a smaller diameter much closer to the stability limit. Although the degree of coherence in the ion cloud will determine the actual ion ejection peak width once mathematical instability is reached, undesirable ion loss and kinetic energy gain is minimized in the simulations of massselective instability by using an appropriate pressure of buffer gas.

\section{Resonant Ejection}

By application of an external field in the $z$ direction, ions that are mathematically stable can be resonantly excited. Therefore they can be ejected at lower rf voltages than is possible with the standard instability scan, thus increasing the mass range of the instrument [22]. The addition of a dipole field in the $z$ direction changes the equations of motion from the Mathieu equation, which is analytically solvable, to a forced Mathieu equation, for which there is no closed-form solution. Numerical integration of Newton's laws of motion is one approximate method available to solve the trajectories of the ions in this type of field.

By approximating the field between the endcaps as a pure dipole, it is possible to simulate the motion of ions during resonance ejection. Figure 4 shows a simulation in which ions are ejected via resonance. Ions of two different mass-to-charge rato values, $\mathrm{m} / \mathrm{z} 202$ and 269, are present and both the normal rf drive (1.1 $\mathrm{MHz})$ field and a supplementary ac $(160.9-\mathrm{kHz}, 6-\mathrm{V})$ field are applied. When the $\mathrm{rf}$ amplitude reaches the resonance point for the on of $\mathrm{m} / z$ 202, namely, $q_{z}=0.4$, the auxiliary field is in resonance with the $z$ direction frequency for this selected ion. However, it is apparent from the simulation that the effects of the supplementary signal are felt on the energy and the axial excursion of the ions of mass 202 long before this point is reached. This is a consequence of the relatively high amplitude of $6 \mathrm{~V}$ chosen for the supplementary ac signal. Under the particular conditions of mitial spatial distribution, buffer gas pressure, and ramp rate used in this simulation, all resonant ions have been ejected by the time the resonance point is reached. Note also that the beating between the main $\mathrm{rf}$ drive and the resonant ac signal is responsible for the pattern seen in the $z$ excursions and in the total kinetic energy of $\mathrm{m} / \mathrm{z}$ 202 in Figure 4. As noted elsewhere [6], a fixed phase relationship between these two signals is desirable to improve performance, but is not normally used. The foregoing factors, especially buffer gas pressure, all influence mass assignment accuracy and mass resolution. The magnitude of the auxiliary field also influ- 


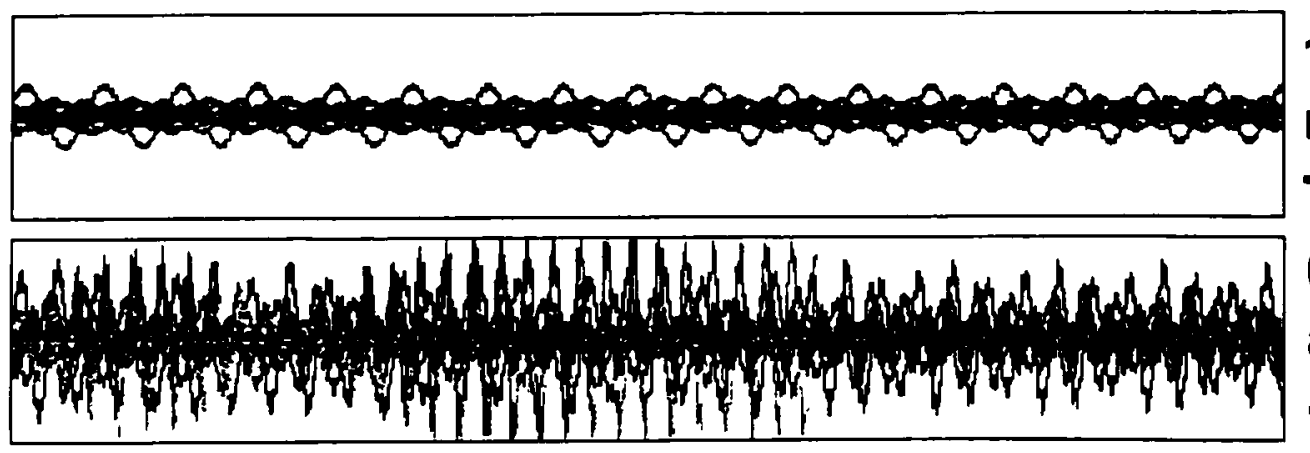

\section{1 [cm] radial $-1$}

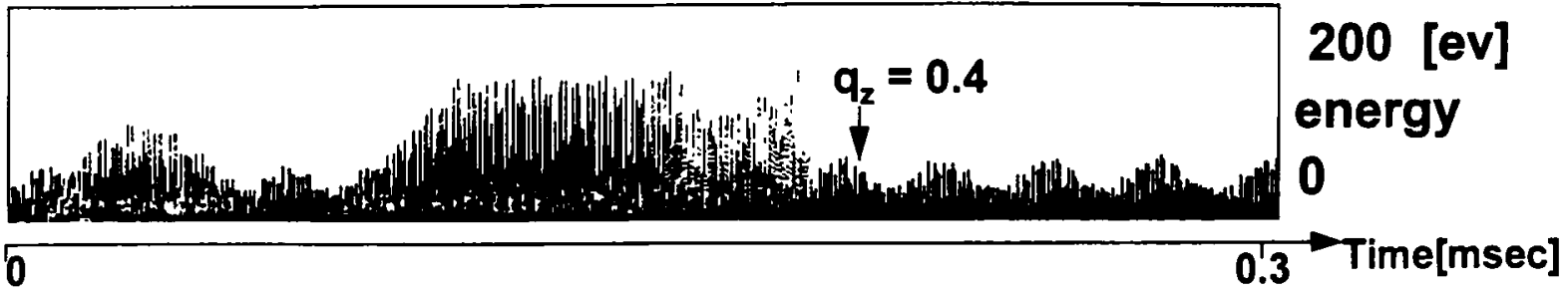

Figure 4. Simulation of resonance ejection in the presence of collision gas. Ions of $m / z 202$ (black) and $m / z 269$ (grey) are considered The on cloud (40 1ons) is initially $2 \mathrm{~mm}$ FWHM and phase angles are randomly distributed. The auxilıary axial frequency is $1609 \mathrm{kHz}, 6 \mathrm{~V}_{0-\mathrm{p}}$ and helium is present at 1 mtorr. The main rf amplitude is scanned from its initial value of $1000 \mathrm{~V}$ to $1165 \mathrm{~V}$, and the theoretical resonance point occurs at $q_{z}=0.4$, which is marked.

ences the processes that occur in the trap. In the presence of helium buffer gas, low amplitude excitation gives ions enough velocity to undergo collisions that can result in an increase in the internal energy of the ion and cause subsequent fragmentation. Higher amplitude excitations can result in direct ion ejection.

The favorable role of the buffer gas to remove kinetic energy and collapse the ion cloud to the center of the trap already has been illustrated, for example, in Figure 3. Its effects during resonance ejection can be deleterious: it can cause spreading in the time over which ions of the same mass-to-charge ratio are ejected (see, however, the section on Poincare plots). This spreading is evident in a resonance ejection experiment in which the rf amplitude is kept constant (not usual in practice) and the resonant ac signal is switched on when desired. The result for a previously cooled and hence tightly bunched ion population is shown in Figure 5a. In this experiment, 20 ions of two masses are simulated. The nonresonant ions of $\mathrm{m} / z 502$ are barely affected by the ac signal, whereas resonant ions of $m / z 69, q_{z}=0.27$, are activated and exit the trap over a narrow range of times. Figure $5 b$ shows the result of the addition of 2 mtorr of helium to the trap. The ions now exit the trap over a greater number of ac cycles, which corresponds to a loss in resolution.

\section{Resonant Activation for Collision-Induced Dissociation}

Collision-induced dissociation in the ion trap normally employs resonant activation of an ion in the presence of buffer gas [12]. It is of interest to estimate the kinetic energy that ions can achieve in these experiments as a
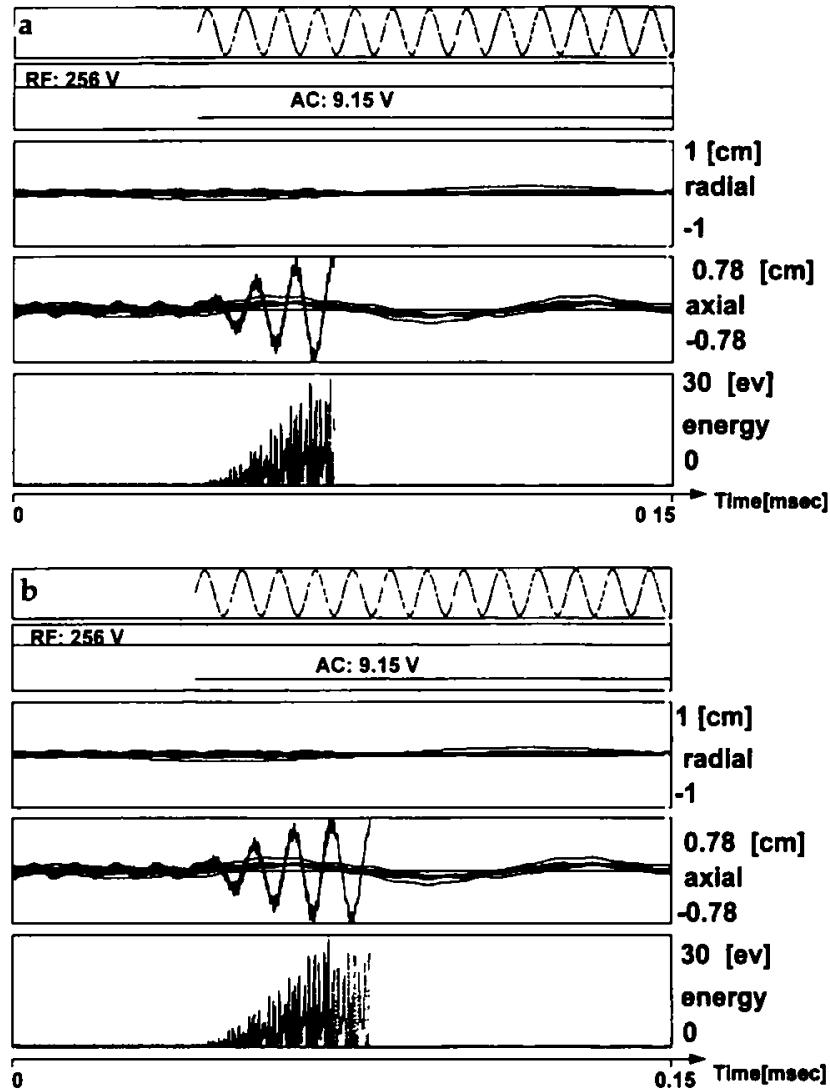

Figure 5. Trapped ions of $m / z 69$ (black) and $m / z 502$ (grey), initially randomly phased and distributed as a sphere of Gaussian intensity (FWHM $0.5 \mathrm{~mm}$ ), are instantaneously exposed to an ac signal of $118.8 \mathrm{kHz}, 9.15-\mathrm{V}$ amplitude. (a) without helium buffer gas $m / z 69$ ions are all resonantly excited and leave the trap in three ac cycles, (b) in the presence of 2-mtorr helium an additional cycle is required. 
function of the amplitude of the resonant ac signal. Information on this point is contained in the simulations shown in Figure 6. This figure shows an experiment performed under strongly activating conditions, namely, $0.7-\mathrm{V}$ ac amplitude and 8 -mtorr pressure. In the early stages of the simulation, the initially large ion cloud contracts rapidly and then expands when the resonant ac signal is applied. The kinetic energy increases to a steady-state value of some tens of electronvolts and multiple collision conditions pertain. Note that these high energies are available only under conditions in which ion ejection from the trap is also quite probable; this contributes to a low yield. The choices of ac amplitude and buffer gas pressure are therefore vital to determine which effect dominates. Note that the conditions illustrated have been carefully chosen so as to maintain the ions with a high kinetic energy for a long period to maximize internal energy deposition in collision-induced dissociation. The axial displacement of the ion population remains roughly constant, whereas the radial motion, which is unaffected by the resonant dipolar field, shows continued cooling throughout the experiment. This type of simulation accounts for the observation that polynuclear aromatic compounds can be dissociated in ion traps, albeit in low yield, to give product ions that are endothermic by $10 \mathrm{eV}$ and more [25].

\section{Simulation of $d c$ Pulse Experiments}

Resonance excitation as just described is routinely used to cause ions to undergo activating collisions with a buffer gas that lead to their fragmentation. This technique is somewhat limited by the fact that very high excitation amplitudes can eject the ion without causing fragmentation. A new experiment, devised to improve internal energy deposition, employs gaseous and/or surface-induced dissociation induced via application of a fast dc pulse [13, 14]. Experiments have been performed with both symmetrical pulses [14], which cause radial excitation, and asymmetrical pulses [13], which cause axial excitation. The fast dc pulses used in these experiments are between 10 and $500 \mathrm{~V}$ in amplitude with a 2-4-ns rise time and they typically last for 2-5 $\mu$ s. To simulate the axial dc pulse experiment, the ITSIM program was modified to replace the periodic function normally associated with the axial auxiliary field with a pulse function $H(t)$. The equation of motion in the $z$ direction therefore changes to

$$
\begin{aligned}
\frac{d^{2} z}{d t^{2}}= & -C(t) \frac{d z}{d t} \\
& +\frac{4 e}{m\left(r_{0}^{2}+2 z_{0}^{2}\right)}(U(t)-V(t) \cos \Omega(t) t) z \\
& +\frac{e}{m 2 z_{0}} V_{\text {aux }}(t) H(t)+\frac{e^{2}}{4 \pi \varepsilon_{0} m} \sum_{1} \frac{1}{\left(r-r_{1}\right)^{2}}
\end{aligned}
$$

Figure 7a shows a simulation of ion motion when a $50-\mathrm{V}$ dc pulse is applied to cause radial acceleration to an ensemble of ions with random phases of motion. The amplitude was chosen to prevent ion ejection; however a nearly instantaneous shift in the center of mass of the ion cloud results, followed by phase coherent motion in both the $x$ and $z$ directions. As was observed in the resonant excitation simulation, ions cannot respond to a resonating signal in less than one

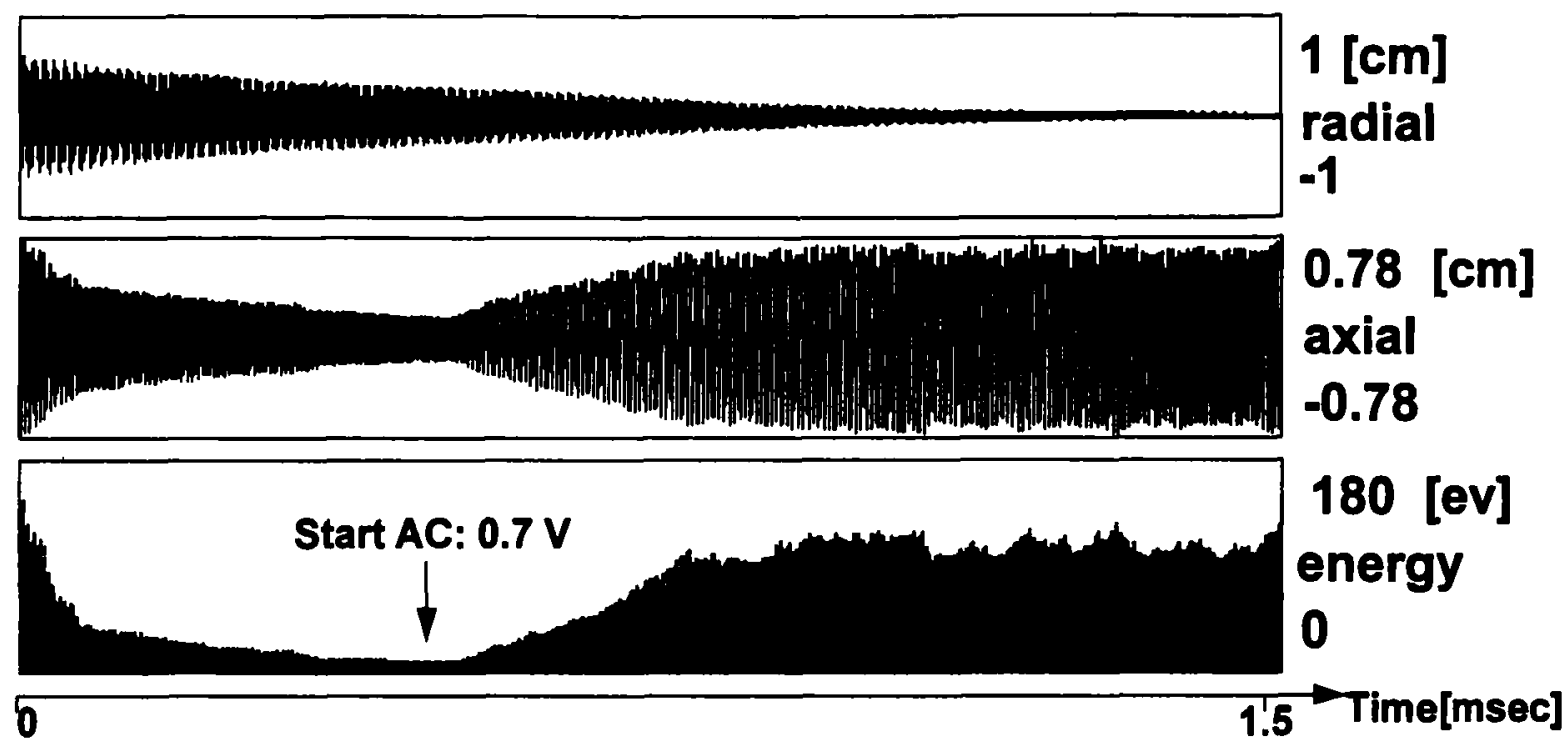

Figure 6. Offsettıng effects of collisional cooling (8-mtorr helium) and resonance heating of 20 ions of $\mathrm{m} / \mathrm{z} 202$ trapped at an $\mathrm{rf}$ amplitude of $1110 \mathrm{~V}$, that $1 \mathrm{~s}, q_{2}=0.4$. Initial conditions: $5-\mathrm{mm}$ Gaussian distribution with random phases The ac was applied after $500 \mu \mathrm{s}$ and had a fixed amplitude of 0.7 $\mathrm{V}$ and frequency $160.9 \mathrm{kHz}$. 

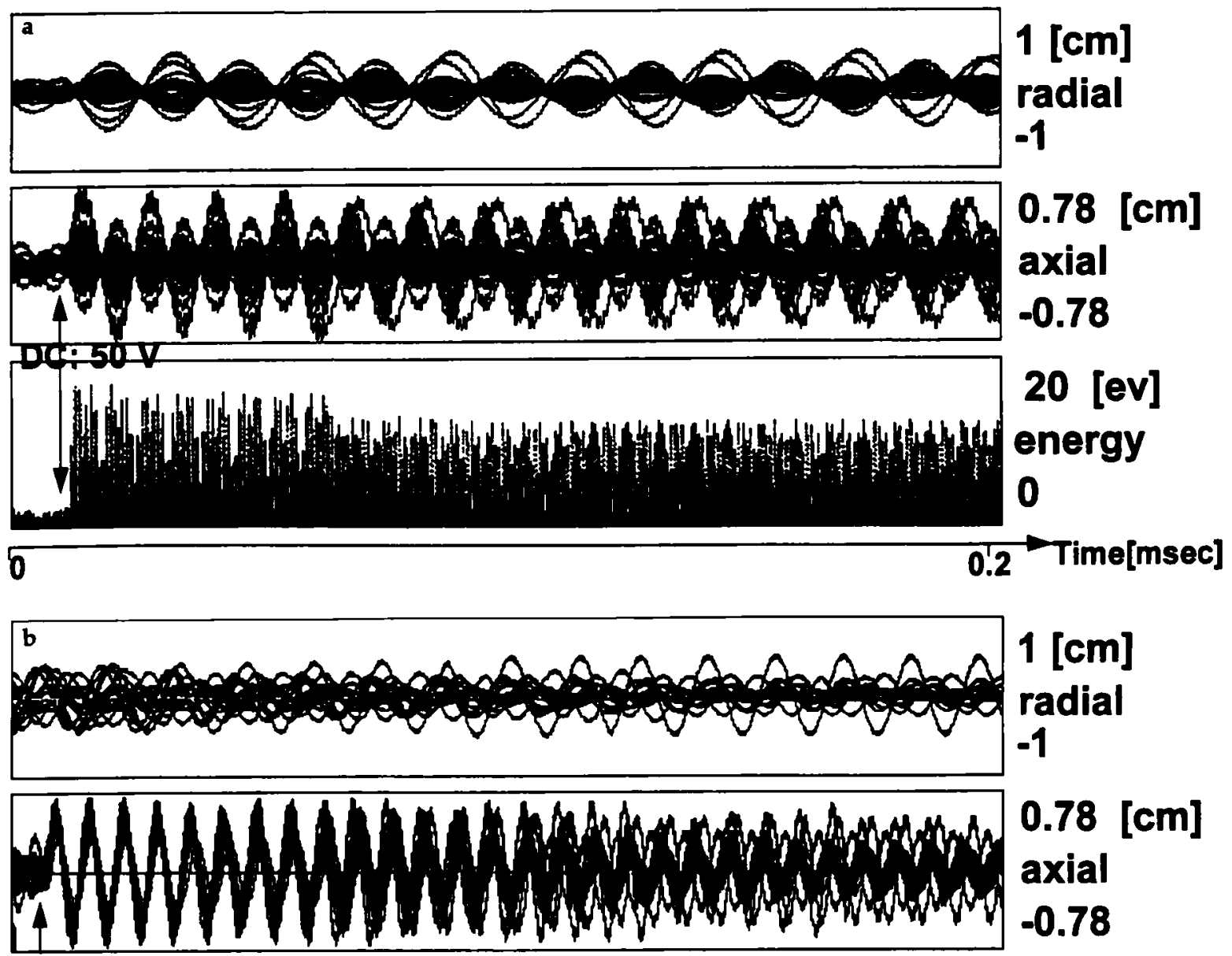

$0.78[\mathrm{~cm}]$

axial

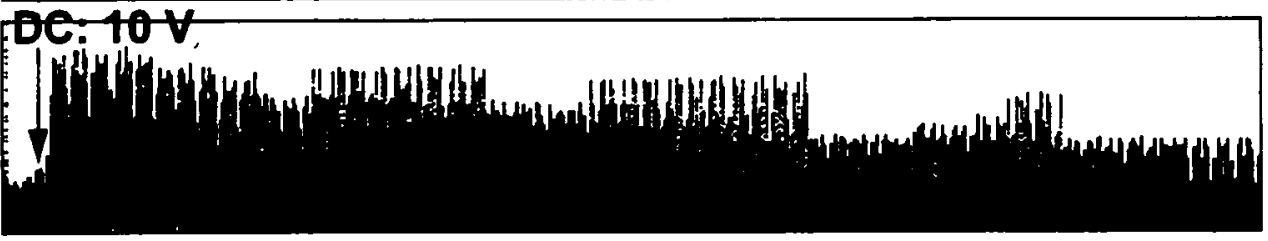

$-0.78$

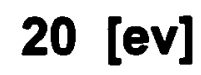

energy

0

\subsection{Time[msec]}

Figure 7. Simulation of ion motion ( $m / z$ 105; $1 \mathrm{~mm}$ FWHM, 20 1ons; $q_{z}=021$ ) excited with (a) a short symmetric dc pulse $(50 \mathrm{~V})$ that produces a quadrupolar field in the axial direction and accelerates ions radially as well as axially in a coherent fashion with 0.2 -mtorr helium as the buffer gas. (b) A short asymmetrical dc pulse $(10 \mathrm{~V})$ that produces a dipolar field and excites ions axially with 1-mtorr neon as the buffer gas

secular cycle; therefore all of the observed activation results from the impulse. Note that the data shown in Figure 7 are for the realistic case in which buffer gas is present in trap. The effect of the buffer gas after some time is to smear the phase of the ions and reduce their kinetic energies and axial excursions. These effects are evident in Figure $7 a$, and become greater at longer times than those shown here.

Figure $7 \mathrm{~b}$ displays results for axial activation with a $10-\mathrm{V}$ pulse. The loss of phase and concomitant reduction in kinetic energy with collisions is evident. Phaselocking is pronounced and in this experiment neon was used as the buffer gas to remove kinetic energy effectively. Note that the stepped appearance of the kinetic energy plot is the result of collisions of single ions with the buffer gas.

It is known that the amount of fragmentation caused by the axial dc pulse is dependent on the pulse amplitude [14]. The multiparticle simulation was therefore used to determine the translational energy and phase coherence achieved by ions in this experiment. To allow an estimate of internal energy deposition, $n$ butylbenzene was used as a thermometer molecule by comparison of the intensity of the $\mathrm{C}_{7} \mathrm{H}_{7}^{+}(\mathrm{m} / z$ 91) and $\mathrm{C}_{7} \mathrm{H}_{8}^{+}(m / z$ 92) fragment ions produced by collisioninduced dissociation. As discussed in ref 14 and the references cited therein, the ratio $91^{+} / 92^{+}$is used as an indication of the amount of internal energy de- 
posited in this molecule. By using the normal resonant excitation method, the highest value for this ratio achieved [12] in an ion trap was 4. By using axial dc pulses, a value of 20 was achieved at large dc pulse amplitudes [14]. Figure 8 reproduces the mass spectra collected in experiments in which the $n$-butylbenzene molecular ion is subjected to $3-\mu \mathrm{s}$ axial dc pulses of increasing amplitude [14]. The pulses were applied when the molecular ion $(m / z \quad 134)$ had a $q z$ value of 0.5 and, as can be seen in Figure 8, the $91^{+} / 92^{+}$ratio increases with pulse amplitude. The results of the simulation of the axial dc pulse experiment are shown as phase space plots in Figure 9. As expected, ions do not appear to undergo resonant excitation due to the
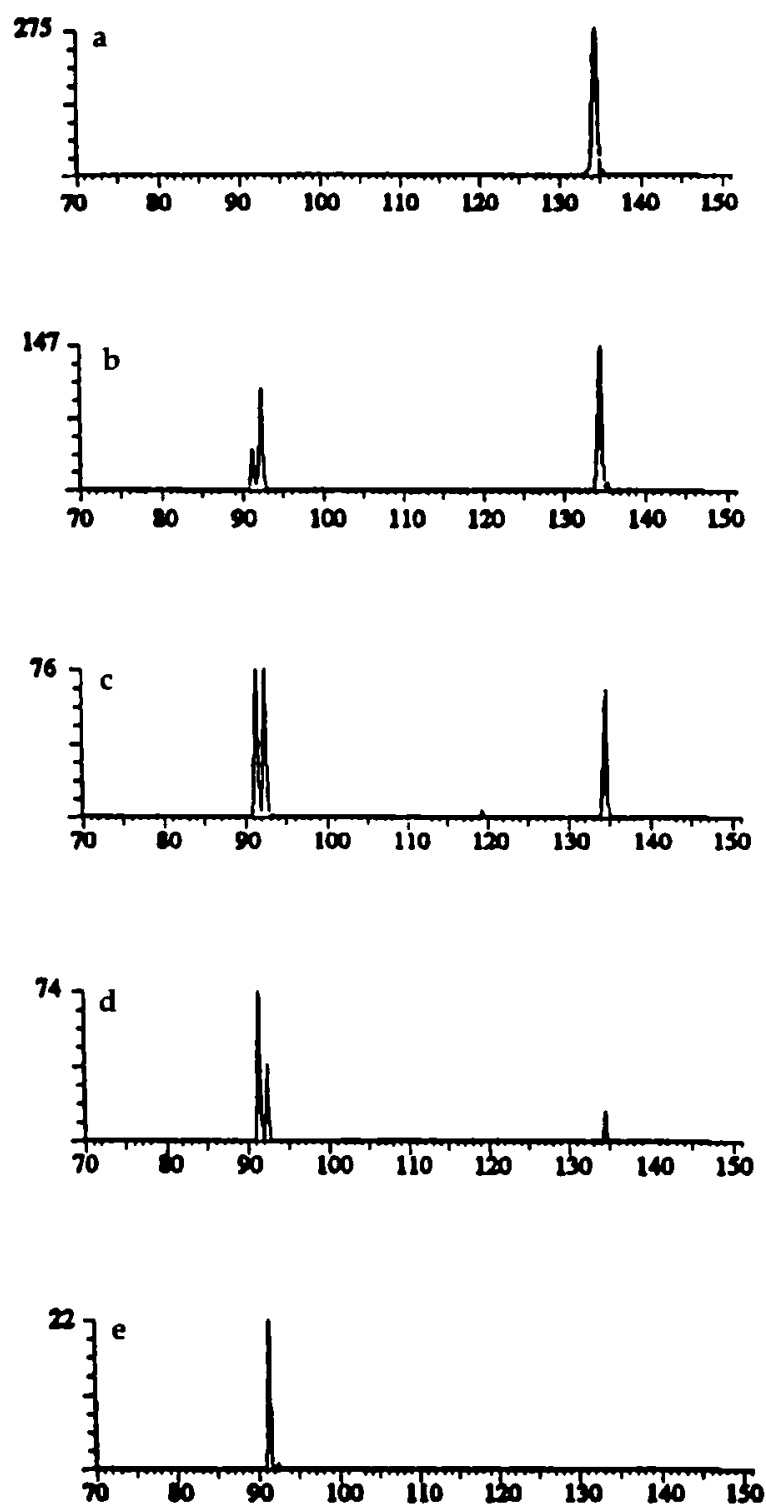

Figure 8. Experimental tandem mass spectra of n-butylbenzene $(m / z$ 134) 1onuzed via electron impact. These data show fragment peaks $\mathrm{C}_{7} \mathrm{H}_{7}^{+} m / z 91$ and $\mathrm{C}_{7} \mathrm{H}_{8}^{+} \cdot m / z 92$ caused by the application of a $3-\mu \mathrm{s}$ dc dipole pulse whule the parent ion was at $q_{z}=0.5$ (from ref 14). (a) Pulse off, no fragmentation occurs due to isolation or storage; (b) pulse voltage $70 \mathrm{~V}$; (c) $104 \mathrm{~V}$; (d) 157 $\mathrm{V}$; (e) $187 \mathrm{~V}$.
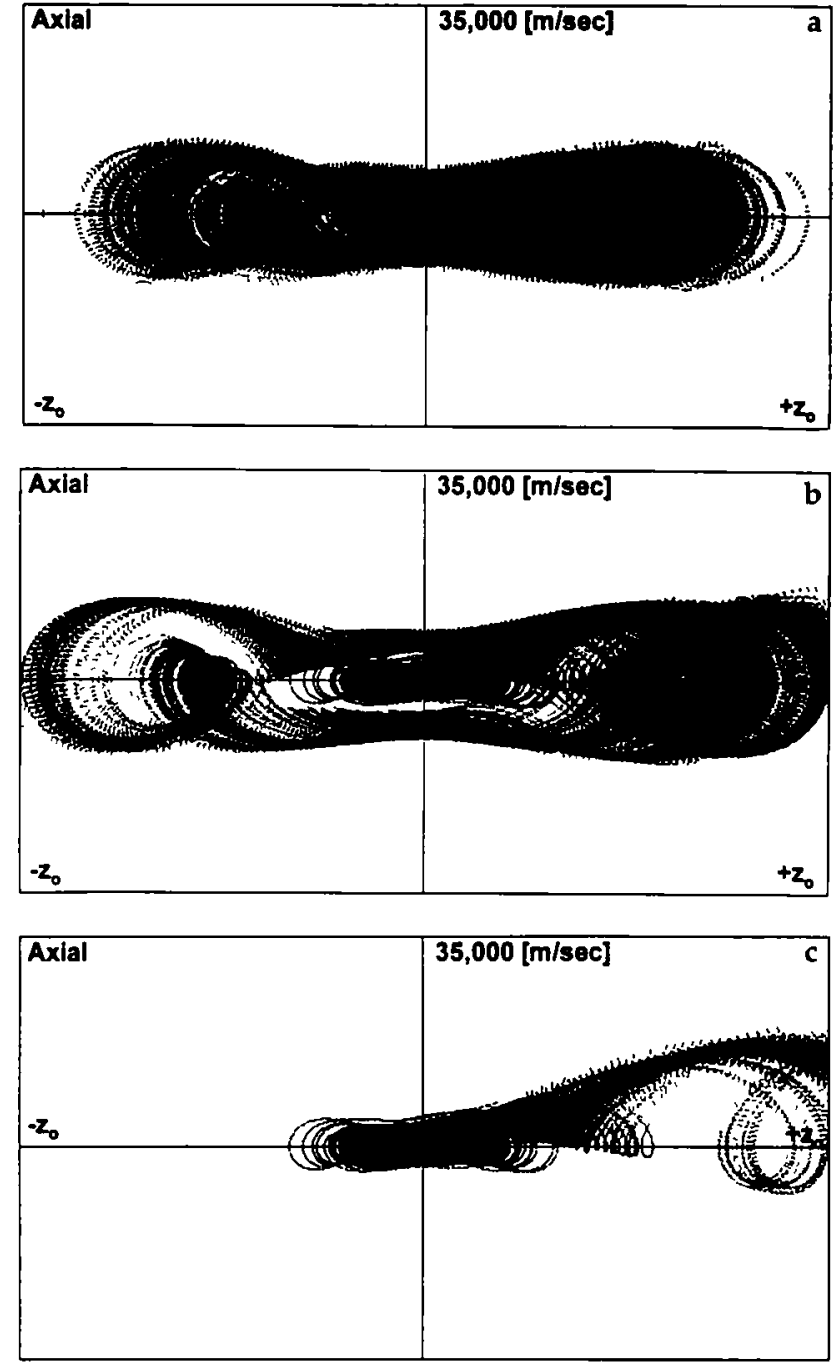

Figure 9. Axial phase-space plots for dipolar dc excitation. The abscissa is the $z$-dimension and the ordinate is velocity The simulation is for 200 ions of $n$-butylbenzene $(m / z 134)$ inutially formed in a cloud FWHM $5 \mathrm{~mm}$ and trapped at $q_{z}=0.5$ in the presence of 1 mtorr of helium All dc pulses were $2 \mu$ s long and amplitudes were (a) $35 \mathrm{~V}$, (b) $50 \mathrm{~V}$, and (c) $80 \mathrm{~V}$

frequency content of the pulse, but rather respond to the steep short-lived gradient. In Figure 9a, the 35-V pulse is shown to axially excite the ions much in the way resonant excitation would, which results in a low $91^{+} / 92^{+}$ratio of 0.7 (compare to experimental data in Figure $8 \mathrm{~b}$ ). Figure $9 \mathrm{~b}$ shows data at a higher pulse amplitudes where the simulations show that some ions undergo surface collisions. Under the conditions of this experiment both surface and gaseous collisions are involved. When the highest dc pulse amplitudes are used (Figure $8 \mathrm{~d}$ and e), a dramatic increase in the $91^{+} / 92^{+}$ratio is observed in the experiment, accompanied by a significant reduction in dissociation efficiency. Both facts are explained by the phase-space plot in Figure 9c, which shows that all the ions strike the electrode and do so with a relatively narrow velocity range $(15,000$ to $20,000 \mathrm{~m} / \mathrm{s}$, i.e., $\sim 210 \mathrm{eV})$, which 
1s sufficiently high to deposit large amounts of internal energy.

The preceding experiments, like almost all others discussed here, were performed both with and without collision gas. As expected for a short time-scale exper1ment, the effects of the buffer gas were small, especially in the high amplitude experiments.

It is of interest to note that the effect of a dc pulse on ion motion, as shown in Figure 7, can be reproduced almost exactly in a simulation that utlizes a short $(5 \mu \mathrm{s})$ supplementary ac pulse of the same frequency to effect activation. This mode of activation is being explored further and will be reported on separately.

\section{Poincaré Sections of Ion Phase Space}

The small scale ITSIM simulation is used primarily to visualize ion trajectories in space as a function of time and as phase-space plots. Trajectory plots are observed either as position traces that track the past position of an ion or as anumation displays of multiple particles within the trap. Animation displays do not lend themselves well to reproduction in this form and are not shown in this paper.

Phase-space plots can be plotted directly (as already shown), or can be simplified by constructing Poincaré sections [26]. From the trajectory plots, the character of the ion motion is visible, whereas examination of the phase-space plot and the Poincare section gives more information on the overall stability and periodic nature of the behavior of the entre collection of ions. For example, in a pure quadrupole field with no collisions, the maximum velocity plotted as a function of ion position corresponds to the Poincare section of stable ion motion. These plots are well known as "stability ellipses" [1,9], and have been quite useful in performing ion injection experiments [27]. Figure 10 gives an example of an axial $(z)$ phase-space plot and a Poincaré section of the phase space, taken at the rf drive frequency of $1.1 \mathrm{MHz}$ and sampled at a zero phase angle for a collection of ions of $\mathrm{m} / \mathrm{z} 202$ in the mass-selective instability scan. The mass-selective instablity experiment gives relatively poor resolution, which is reflected un the dispersion seen in the Poincare section. Note that the points that lie further from the origin occur later in the simulation time.

In the mass-selective instability scan, ions are ejected by forcing those of increasing mass-to-charge ratio to acquire unstable trajectories by ramping the $\mathrm{rf}$ voltage. The stability limit can be stated in terms of $q_{z}\left(q_{z}=\right.$ 0.908 ), which relates the voltage required to eject an ion to its mass-to-charge ratio. It has been observed that the mass resolution of the mass-selective instability scan can be improved greatly by performung resonant ejection, either near the stability limit ("axial modulation") [28] or much below the stability limit [12]. The mass resolution obtained by combining slow rf scans with resonant ejection is extremely high-in
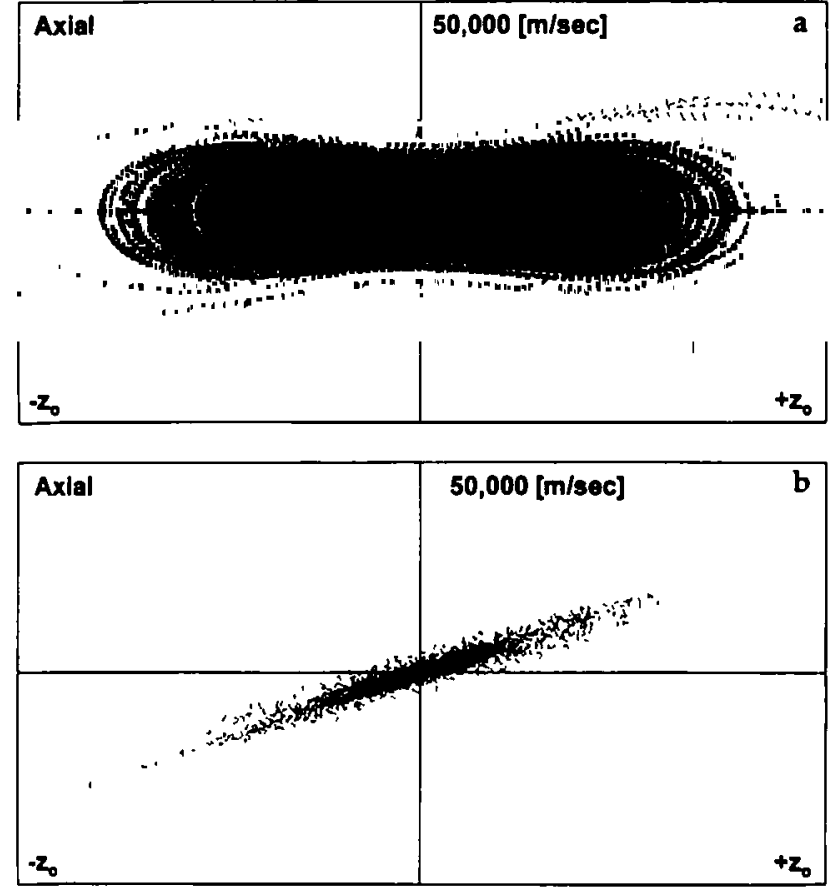

Figure 10. (a) Axial phase space and (b) Poincaré section for 20 ions of $m / z 202$ in a mass-selective instability scan in the presence of helium at $8 \mathrm{mtorr}$

the range of $10^{6}$ and above $[29,30]$. Some explanations for the improved resolution can be gleaned by comparing Poincaré sections for resonant ejection and conventional mass-selective instability scans. When ions of $\mathrm{m} / \mathrm{z} 202$ are bought into resonance $\left(q_{z}=0.4\right)$ by applying $\sim 2520 \mathrm{~V}_{0-\mathrm{p}}$ to the ring electrode in the presence of a buffer gas, the motion is stabilized withun the electrode structure, despite mathematical instability. The trajectory plots for the $x$ and $z$ directions (Figure 6) show the balance between energy loss and energy gain. The axial Poincare section (Figure 11) shows two connected ellipses. one of small dimension is reached after initial cooling of the ion cloud by collisions and a larger ellipse into which the ions are driven later in the simulation period shown in Figure 6. The tightly constrained energy-position relationship seen in this plot is also characteristic of slow scan resonance ejection experiments and is responsible for their high resolution qualities. A limit cycle is created toward which all ions are driven. In phase space, once two trajectories fall on the same limit, they are phase-locked. This limit cycle forces the ions to move as a coherent ion cloud with a very narrow range of maximum amplitudes and phase shifts. As the ion absorbs energy from the auxiliary field, the limut cycle will increase in diameter until the electrode dimension is reached and the ions are ejected. If the ions are given time to adjust to changes in the rf amplitude, the limut cycle will remain a very narrow ellipse. When the rf ramp is slow, ions are allowed to equilibrate and to maintain their phase coherence, which results in the high mass resolution observed when slow rf ramps are used. 

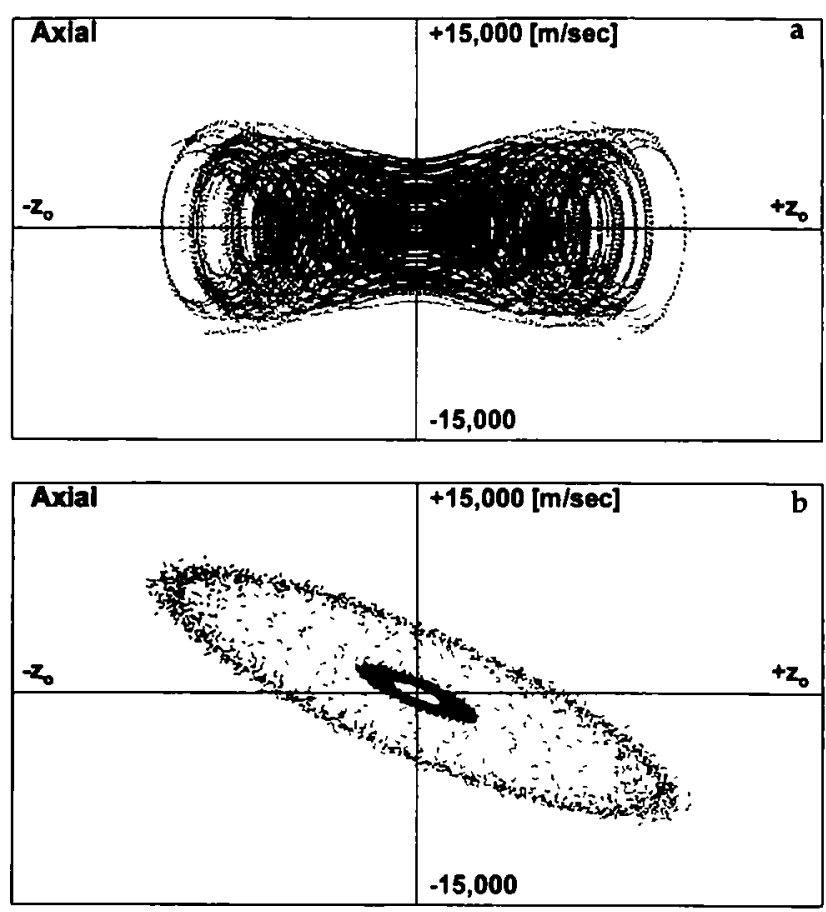

Figure 11. (a) Axial phase space and (b) Pouncaré section for a resonant ejection experiment in the presence of buffer gas in which a limit cycle is reached The simulation is for 20 ions of $m / z 202$ at $q_{z}=04$, excited by an ac signal of $1609 \mathrm{kHz}, 07 \mathrm{~V}$, and deexcited by 8-mtorr helium (a) 06 -ms run thmes, (b) 1.5-ms run time.

The Poincare sections for mass selective instability scans (Figure 10), on the other hand, indicate that ions of the same mass-to-charge ratio value with only slightly different initial conditions will have extremely different trajectories that lead to a wide range of ejection times and hence to the observed low mass resolution.

\section{Conclusions}

Multiparticle simulations allow ranges of motion to be observed for ions of single mass-to-charge ratios and allow the direct comparison of the motion of ions with different mass-to-charge ratios (features not readıly shown in the single-color plots used here). The program also contains capabilities for the study of ion-1on interactions, results for which do not become apparent in small scale simulations like those shown here. Visualization of ion motion in several different formats permits the characterization of ion motion during excitation. Poincaré sections have been added to the tools previously available to analyze ion motion via computer simulation. In the absence of collisions, stable motion can be recognized as stable elliptical patterns in the Poincare section.

Collisions with buffer gas during ion excitation are demonstrated to have a large effect, not only on the energy ranges obtained in dampened systems, but also on the character of the ion motion. Collisions during the mass-selective instability scan can have a beneficial effect on peak amplitude and mass resolution. On the other hand, collisions tend to dephase ions already moving coherently and their effect in the resonance ejection experiment can cause loss of resolution. In spite of this, it is also possible to use collisions to force a population of ions into coherent motion in a resonant ejection experiment.

Examination of the Poincare section reveals whether motion is stable, or random or chaotic. In contrast to the uncoordinated motion of the ions during the mass-selective instability scan, stable motion is evident during damped resonant excitation. In this case the ion motion can approach a limit cycle in phase space, which represents an equilibrium between the dampening effects of the buffer collisions and the excitation caused by the external field. In this case, ions become phase-locked, which generates a condition in which ions with large differences in phase and maximum amplitude converge on a narrow range of phase angles and maximum amplitude. The result is much higher resolution in the resonant ejection case due to the narrow range of maximum amplitude and the uniform increase in maximum amplitude for all ions in resonance. This result also indicates why slow scan rates have narrow ion ejection peak widths. The peak width of an ion ejection event is controlled by the distribution of ton maximum amplitudes and ion phase shifts. If the 1ons are allowed to equilibrate between the excitation energy and the buffer collisions, the narrow distributions persist. If the scan is too fast, the equilibrium is not obtained and a broad range of phase angles and maximum amplitudes will exist for a single massto-charge ratio, which leads to a wider ejection peak.

\section{Acknowledgments}

This work was supported by the National Science Foundation (CHE87-21768) and Finnigan MAT through the Chemistry Department Industrial Associates Program The work of Hans-Peter Reiser, the author of the ITSIM program, is gratefully acknowledged RKJ acknowledges support through a fellowship provided by Elı Lilly and Company Computer facilities were provided by the Purdue University Computer Center on the VAX 8800 through the Presidential Computing Reserves The assistance of Steve Lammert, Kathy Cox, Lynn Horn, Jon Willams, and Curt Cleven is acknowledged.

\section{References}

1. March, R. E.; Hughes, R. J. Quadrupole Storage Mass Spectrometry, Chemical Analysis Series; Wiley' New York, 1989, Vol 102.

2. Todd, J F. J, Mass Spectrom. Rev, 1991, 10, 3.

3. March, R. E., McMahon, A W , Londry, F. A., Alfred, R. L., Todd, J. F. J.; Vedel, F. Int I Mass Spectrom Ion Processes 1989, 95, 119.

4. March, R. E., McMahon, A W, Allıson, E. T, Londry, F. A, Alfred, R L.; Todd, J. F J., Vedel, F Int. I Mass Spectrom Ion Processes 1990, 99, 109 
5. March, R. E., Londry, F A, Alfred, R. L., Todd, J F J, Penman, A D., Vedel, F., Vedel, M Int I Mass Spectrom Ion Processes, 1991, 110, 159

6. Julıan, R K., Reiser, H.-P, Cooks, R G. Int / Mass Spectrom Ion Processes 1993, 123, 85

7. Reiser, H.-P, Julıan, R. K., Cooks, R G Int I Mass Spectrom Ion Processes 1992, 121, 49.

8. Dawson, P. H., Lambert, C Int / Mass Spectrom Ion Phys $1975,16,269$

9 Dawson, P H Quadrupole Mass Spectrometry and its Applicahonts, Elsevier Amsterdam, 1976.

10 Reiser, H.-P Ph.D Thesis, Unuversity of Geissen, 1992

11 Stafford, G C, Kelley, P E, Syka, J E P, Reynolds, W F, Todd, J. F J Int J Mass Spectrom lon Processes 1984, 60, 85

12 Louns, J N, Cooks, R. G, Syka, J E P, Kelley, P E, Stafford, G C, Todd, J F J Anal Chem 1987, 59, 1677

13 Lammert, S A, Cooks, R G I Am Soc Mass Spectrom 1991, 2,487

14 Lammert, S A, Cooks, R. G Raptd Commun Mass Spectrom 1992, 6, 528

15. Lammert, S A Ph D. Thesis, Purdue Unuversity, 1992.

16. Goennger, D. E, Whitten, W B, Ramsey, J. M , McLuckey, S A, Glish, G. L Anal Chem 1992, 64, 1434

17 Kuight, R D Int / Mass Spectrom Ion Phys 1983, 51, 127

18 Louris, J N, Schwartz, J C, Stafford, G C, Syka, J E P., Taylor, D Proc 40th ASMS Conference on Mass Spectrometry and Alled Topics, Washington, DC, 1992, p 1003
19. McDanuel, E W. Collision Phenomena m lonized Gases, Wiley New York, 1964, p 5

20 McDaniel, E W Collision Phenomena m lonized Gases, Wiley New York, 1964, p 18

21. Lourss, J N, Brodbelt-Lustig, J S ; Cooks, R. G., Glish, G L, Van Berkel, G J, McLuckey, S A Int J Mass Spectrom Ion Processes 1990, 96, 117

22 Kaiser, Jr, R E, Cooks, R. G, Stafford, G C, Syka, J E P., Hemberger, $\mathrm{P} H$ Int I Mass Spectrom lon Processes 1990, 106,79

23. Schwartz, J C, Syka, J E P., Jarıne, I J Am Soc Mass Spectrom 1991, 2, 198

24 Kaıser, R. E, Cooks, R G; Syka, J E P, Stafford, G C Rapıd Commun Mass Spectrom 1990, 4, 30.

25 Nourse, B D.; Cox, K A, Morand, K L, Cooks, R G. I Am Chem Soc 1992, 114, 2010.

26 Moon, F C Chaotic Vibrations, Wiley New York, 1987 For a treatment of parametrically driven oscillators that have Mathieu form, see pages 79-81

27 Louris, J N, Amy, J. W, Rudley, T Y, Cooks, R G Int I Mass Spectrom Ion Processes 1989, 88, 97

28 Weber-Grabau, M, Kelley, P E, Bradshaw, S C, Hoekman, D J Proc 36th ASMS Conference on Mass Spectrometry and Alled Toptes, San Francisco, CA, 1988, p 1106

29 Schwartz, J C, Syka, J E P, Jardıne, I I Am Soc Mass Spectrom 1991, 2, 198

30 Londry, F. A, Wells G J, March, R E Rapid Commun Mass Spectrom 1993, 7, 43 